\title{
In vitro antibacterial activity of telithromycin against Enterococcus spp. isolated from China
}

\author{
Yanpeng Xiong \\ Shenzhen University \\ Junwen Chen \\ Shenzhen University \\ Xiang Sun \\ Shenzhen University \\ Guangjian Xu \\ Shenzhen University \\ Peiyu Li \\ Shenzhen University \\ Qiwen Deng \\ Shenzhen University \\ Zhijian Yu \\ Shenzhen University \\ Zhong Chen \\ Shenzhen University \\ Jinxin Zheng ( $\sim$ jinxinzheng@fudan.edu.cn) \\ Shenzhen University
}

Research article

Keywords: Telithromycin, Enterococcus faecalis, Enterococcus faecium, Resistance, Biofilm

Posted Date: May 1st, 2020

DOI: https://doi.org/10.21203/rs.3.rs-24213/v1

License: (c) (i) This work is licensed under a Creative Commons Attribution 4.0 International License. Read Full License 


\section{Abstract}

Background: Enterococci has resistant to a wide range of antimicrobials, and the treatment of enterococcal infection has always been an issue of concern. This study aimed to explore the new ketolides antimicrobials-telithromycin, against the planktonic cells and biofilms of enterococci. The minimum inhibitory concentration (MIC) of telithromycin was determined. The sequence types (STs) and genotypes of resistance to erythromycin in enterococci were detected. Furthermore, the effect of telithromycin against the biofilms of enterococci were investigated.

Results: A total of 280 Enterococcus faecalis and 122 Enterococcus faecium isolates were collected from individual inpatients in China. Telithromycin showed excellent activity against the $E$. faecalis and $E$. faecium strains no matter sensitive or resistant to erythromycin with erm $A$, erm $B$ or erm $C$, with the $\mathrm{MIC}_{50}$ at $2 \mu \mathrm{g} / \mathrm{mL}$ and $4 \mu \mathrm{g} / \mathrm{mL}$, respectively. The predominant STs of E. faecalis isolates were ST16, ST30, ST179, and ST18, ST78, ST80 were the predominant STs of E. faecium isolates. Moreover, 87.1\% (135/158) and 80.4\% (41/51) isolates of the predominant STs carried the erm genes in $E$. faecalis and $E$. faecium isolates, respectively. The subinhibitory concentration of telithromycin (at $1 / 4 \times$ and $1 / 8 \times$ MICs) significantly inhibited the biofilm formation of $16 \mathrm{E}$. faecalis isolates. Telithromycin (at $8 \times \mathrm{MIC}$ ) indicated the removal effect on the established biofilms of 8 E. faecalis isolates, and combined with ampicillin eradicated more biofilms than telithromycin or ampicillin alone.

Conclusion: Telithromycin showed an excellent activity against the planktonic cells of $E$. faecalis and $E$. faecium, and against the biofilms of $E$. faecalis.

\section{Background}

\section{Enterococci are common nosocomial infections recognized as opportunistic pathogens}

and form biofilms, being associated with urinary tract infection, wound infection, intra-abdominal and pelvic regions infection, and bloodstream infection [1-3]. Enterococcus faecalis is the most common species of enterococcus, accounting for $85 \%$ to $90 \%$ of human enterococcal infections [4]. Enterococcus faecium accounts for most of the rest of infections induced by enterococci [5]. According to previous reports, biofilms are recognized as a major cause of nosocomial infections, accounting for more than $80 \%$ microbial infection, and both of $E$. faecalis and $E$. faecium are able to form biofilms [1, 6]. Compared to planktonic cells, enterococci in biofilms are more extremely resistant to antimicrobial agents [7]. Moreover, enterococci have emerged increasingly resistant to antimicrobials, and the treatment of enterococcal infection has always been an issue of concern. Furthermore, it has been discovered that enterococcus frequently resistant to oxazolidinones, glycopeptides, aminoglycosides, $\beta$-lactams, and macrolides [8, 9]. Therefore, there is an urgent need to assess any new therapeutic alternative treatment for enterococcal infections.

Telithromycin (HMR3647), known as a member of ketolides antibiotic, was approved by the US Food and Drug Administration (FDA) for the treatment of community-acquired pneumonia (CAP), acute exacerbations of chronic bronchitis (AECB), and acute maxillary sinusitis (AMS) [10]. The resistance to macrolide-lincosamide-streptogramin B (MLSB) in enterococci based on mono- or bimethylation of 23S rRNA and its target sites modification (A2058 or A2509) on domain V [11-13]. Methylation identified as usually dominated by the acquisition of erm genes, which encode methyltransferases is specific adenine residues of N6-dimethylation in the conserved region of rRNA [14, 15]. However, telithromycin has one more target site modification (A725) on domain $₫$ compared with MLSB antibiotics, including erythromycin, and resulting in affinity increased for rRNA and impairing the function of methylate, and telithromycin is capable of remaining bound and active $[15,16]$.

In this study, we examined the in vitro antibacterial activity of telithromycin against enterococcal clinical isolates collected from inpatients in Shenzhen Nanshan People's Hospital and compared with other antimicrobials. The effect of telithromycin against the biofilms of E. faecalis isolates was also explored in this study.

\section{Results}

\section{In vitro activity of telithromycin against enterococci isolates}

The E. faecalis isolates ( $n=280)$ were retrospectively collected from urine $(n=129,46.1 \%)$, tracheal secretions $(n=48,17.1 \%)$, blood $(n=29,10.4 \%)$, bile $(n=19,6.8 \%)$, phlegm $(n=9,3.2 \%)$ and others sources $(n=46,16.4 \%)$ (Fig. S1. A). A total of 122 E. faecium isolates were also collected form urine ( $n=59$, 48.4\%), tracheal secretions ( $n=20,16.4 \%)$, blood $(n=16,13.1 \%)$, bile $(n=12,9.8 \%)$ and other sources $(n=15,12.3 \%)$ (Fig. S1. B). The enterococcal isolates were highly resistant to erythromycin (ERY) and common tetracycline antibiotics, including tetracycline (TET), doxycycline (DOX), minocycline (MIN), and were more sensitive to vancomycin (VAN), nitrofurantoin (NIT), and linezolid (LZD) (Table 1 and Table 2).

Telithromycin showed an excellent activity against the E. faecalis and E. faecium strains, with the $\mathrm{MIC}_{50} / \mathrm{MIC}{ }_{90}$ at the $2 / 8 \mu \mathrm{g} / \mathrm{mL}$ and $4 / 8 \mu \mathrm{g} / \mathrm{mL}$, respectively (Table 1 and Table 2). It should be noted that the E. faecalis isolates were susceptible to ampicillin (274/275) (Table 1), on the contrary, the E. faecium isolates exhibited high resistance to ampicillin (98/112) (Table 2). E. faecalis and the E. faecium isolates had a higher $\mathrm{MIC}_{50} / \mathrm{MIC}_{90}$ to $\mathrm{ERY}$ $(>256 />256 \mu \mathrm{g} / \mathrm{mL}$ and $>256 />256 \mu \mathrm{g} / \mathrm{mL}$ ). It was noteworthy that 212 E. faecalis isolates with ERY MIC $\geq 8 \mu \mathrm{g} / \mathrm{mL}$ (resistance rate reached at $75.7 \%$ ), but only 33 isolates with telithromycin MIC $\geq 8 \mu \mathrm{g} / \mathrm{mL}$ (Table 1).

\section{In vitro activity of telithromycin with ERY-specific resistance genes}


To examine the effect of ERY-specific resistance genes on susceptibility of telithromycin in E. faecalis and E. faecium isolates, PCR assays were performed to detect the expression of erm A, erm B, erm C (Table 3 and Table 4). The data showed that telithromycin $\mathrm{MIC}_{50 / 90} \mathrm{values}$ of $2 / 8 \mu \mathrm{g} / \mathrm{mL}$ and $4 / 8 \mu \mathrm{g} / \mathrm{mL}$ were obtained respectively in $E$. faecalis and $E$. faecium isolates with the ERY-specific resistance genes, suggesting that the presence of resistance genes may affect telithromycin sensitivity of enterococcal isolates. However, the ERY sensitivity rate of enterococcal isolates harboring erm $A$, erm $B$ or erm $C$ genes reached a lower level at 0-10\% (Table 3 and Table 4).

\section{MICs of telithromycin and ERY to MLST and carriage of erm genes}

MLST was used to determine the ST distribution of the E. faecalis and E. faecium isolates (Table S1 and Table S2). The present research detected fortyfour STs in E. faecalis isolates, with the predominant STs being ST16, ST30 and ST179, and 44 isolates of new ST that were not recorded in MLST database. Moreover, sixteen STs in E. faecium isolates, with the predominant STs being ST18, ST78 and ST80, and 14 isolates of new ST were found in this study.

The distribution of telithromycin and ERY MICs with the predominant STs in E. faecalis and E. faecium isolates as shown in Table 5 and Table 6 . The data indicated that 26.6\% (42/158), 9.5\% (15/158), 47.5\% (75/158), 16.4\% (26/158) and 15.7\% (8/51), 2\% (1/51), 23.5\% (12/51), 58.8\% (30/51) of the $E$. faecalis and $E$. faecium isolates with telithromycin $\mathrm{MIC} \leq 1,2,4, \geq 8 \mu \mathrm{g} / \mathrm{mL}$, respectively. However, the ERY non-sensitivity rate of the $E$. faecalis and $E$. faecium isolates with the predominant STs reached a higher level at 98.7\% (156/158) and 94.1\% (48/51), respectively. Moreover, 87.1\% (135/158) and $80.4 \%(41 / 51)$ isolates of the predominant STs carried the erm genes in E. faecalis and E. faecium isolates, respectively (Table 5 and Table 6). Our results demonstrate that telithromycin may exert more excellent in vitro antimicrobial activity against enterococcus than ERY.

\section{The effect of telithromycin against the biofilms of $E$. faecalis isolates}

To determine the inhibitory effect of telithromycin on biofilms of sixteen E. faecalis isolates, biofilm assays were performed in 96 well microtiter plates by detecting the OD570 after crystal violet staining, and 1/2x, 1/4x and 1/8x MICs were used to in this study. As shown in Fig.1, telithromycin dramatically inhibited the biofilm formation of sixteen E. faecalis isolates that MIC values as listed in Table S3. Moreover, we investigated the eradicated effect of telithromycin on established biofilms of 4 E. faecalis isolates and compared with common antimicrobials of AMP, VAN, LZD (Fig. S2). The data showed that telithromycin and AMP were capable of eradicating the established biofilms of E. faecalis, but VAN and LZD could not. Thus, we further determined the eradicating effect of telithromycin combined with AMP on the established biofilms of 8 E. faecalis isolates, suggesting that telithromycin exhibited robust synergistic effect with AMP (Fig. 2).

\section{Discussion}

Enterococci species are commensals bacteria in human gastrointestinal tract with the ability to cause various nosocomial infections. They are not only equipped with various intrinsic antimicrobial resistances, but also are able to acquire mutation and/or new resistance genes [17, 18]. Telithromycin (HMR3647), known as a novel ketolide antimicrobial agent applied for treatment of respiratory infection [19]. Wolter $\mathrm{N}$ et al. identified telithromycin resistance in Streptococcus pneumoniae is associated with a deletion in the crucial sequence of rRNA methylation induced by erm B [20]. Uzun B et al. reported that telithromycin resistance in erythromycin-resistant isolates, but all erythromycin-susceptible isolates were also susceptible to telithromycin [21]. However, the treatment of telithromycin for enterococci infection has been studied around the world, and its effect is better than those of the first and second generation macrolides [22]. In this study, our data suggested that the E. faecalis and E. faecium isolates had a lower $\mathrm{MIC}_{50} / \mathrm{MIC}_{90}$ of telithromycin at the $2 / 8 \mu \mathrm{g} / \mathrm{mL}$ and $4 / 8 \mu \mathrm{g} / \mathrm{mL}$, respectively. However, ERY $\mathrm{MIC}_{50} / \mathrm{MIC}_{90}$ was $>256 />256 \mu \mathrm{g} / \mathrm{mL}$ and the resistance rate was reached at more than $75 \%$ in both of the E. faecalis and E. faecium isolates. Since increasing evidence has suggested that the prevalence of AMP, VAN, and LZD resistance in enterococci around the world $[18,23,24]$. Our results were consistent with that the emergence of AMP resistance among $E$. faecium isolates in our hospital (resistance rate at 87.5\%). However, those of ERY-resistant and ERY-non-resistant $E$. faecalis isolates were highly sensitive to AMP, VAN and LZD. These results suggest that telithromycin is more effective than ERY, and both antibiotics of VAN and LZD may be more suitable for the treatment of enterococcal infections in our hospital.

The macrolide-lincosamide-streptogramin B resistance phenotype widely appears in erythromycin resistance clinical strains. Weisblum B et al. demonstrated that erythromycin resistance in Staphylococcus aureus is section of the macrolide-lincosamide-streptogramin B resistance phenotype after erythromycin was used in clinical practice [25]. This phenotype was determined by erm gene that product an erythromycin resistance methylase, inducing methylation of specific adenosine residues on the 23S rRNA, which causes resistance of newly synthesized ribosomes to macrolidelincosamide-streptogramin B antimicrobials $[14,15]$. Sule Celik et al. described that the emergence of high-level erythromycin resistance may lead to poorer prognosis, more severe recurrence, and higher mortality, in enterococcal infections [26].

In this study, erm genes were examined and revealed that erm $B$ gene was the most common resistance genes in ERY-resistant isolates of $E$. faecalis. However, both of erm $B$ and erm $C$ gene were detected, which are the most common resistance of genes in $E$. faecium isolates. These results suggest that in vitro antibacterial activity of telithromycin is associated with ERY-specific resistance genes.

Recombination is involved in genetic variation of resistance and virulence determinants, which might promote hospital adaptation among enterococcus bacterial including E. faecalis and the E. faecium [27, 28]. Ruiz et al. investigated that ST16 is composition of hospital-adapted strains, suggest that most continual alteration in clinical isolates of enterococcal, and promoting to generate multiple-resistance, consequently [27]. In the present study, fortyfour STs were detected in E. faecalis isolates, with the absolutely predominant STs being ST16 and ST179, and they are belonging to clonal complex of

Page 3/11 
CC16. Thus, the treatment of antibiotics for $E$. faecalis infection should choose reasonably according to the characteristics of CC16. Moreover, sixteen STs were determined in E. faecium isolates, with the positively predominant STs being ST18 and ST78. Comparison of other STs, the susceptibility of predominant STs to telithromycin is not well in enterococci isolates. Furthermore, the carriage of erm genes in enterococcal isolates with the predominant STs were confirmed, suggesting that the sensitivity of isolates of predominant STs to telithromycin may be affect by erm genes in enterococci.

Increasing evidence has manifested that biofilms formation is play a crucial role in enterococcal infection [29]. E. faecalis is capable of forming biofilms on inanimate and living surfaces, and it promotes tolerance and reduces sensitivity to environmental influences or antimicrobial measures [30, 31]. Thus, targeting biofilms formation of $E$. faecalis can potentially contribute to the treatments for enterococcal infection. As previously described, telithromycin is able to decrease and eradicate Staphylococcus aureus biofilms [32]. Our results showed that telithromycin was dramatically inhibited the biofilm formation of sixteen E. faecalis isolates, and exhibited excellent eradicated effect combined with AMP on the established biofilms of eight $E$. faecalis isolates. However, telithromycin decreased biofilms formation of $E$. faecalis isolates was not dose-dependent. Further investigation is needed to characterize the inhibitory effect of telithromycin on biofilms formation of E. faecalis.

\section{Conclusion}

In summary, telithromycin showed robust in vitro antimicrobial activity against enterococci isolated clinically from Shenzhen Nanshan People's Hospital with lower $\mathrm{MIC}_{50 / 90}$ values than ERY. Moreover, telithromycin exhibited excellent activity of E. faecalis and E. faecium isolates with the ERY-specific resistance genes at $\mathrm{MIC}_{50 / 90}$ values of $2 / 8 \mu \mathrm{g} / \mathrm{mL}$ and $4 / 8 \mu \mathrm{g} / \mathrm{mL}$. We further demonstrated that telithromycin could repress $E$. faecalis biofilms formation, eradicate the established biofilms and exhibit robust synergistic effect combined with AMP. These results suggest that telithromycin is an efficient antimicrobial agent for enterococcus.

\section{Methods}

\section{Bacterial isolates and growth condition}

A total of 280 non-duplicate E. faecalis and 122 non-duplicate E. faecium strains were isolated from individual patients at Shenzhen Nanshan People's Hospital from 2011-2015. Bacterial isolates were determined by standard methods using a VITEK 2 system (Biomérieux, Marcy l'Etoile, France). E. faecalis ATCC29212 and OG1RF (ATCC 47077) were tested as quality control strains. The isolates were cultured in TSB (Oxoid, Basingstoke, UK) at $37^{\circ} \mathrm{C}$ with a shaker at $220 \mathrm{rpm}$. The antimicrobials telithromycin (TEL), erythromycin (ERY), ampicillin (AMP), vancomycin (VAN), tetracycline (TET), doxycycline (DOX), minocycline (MIN), ciprofloxacin (CIP), nitrofurantoin (NIT), linezolid (LZD), tedizolid (TED) and tetracycline (TEC) were purchased from MCE (Princeton, NJ).

All procedures involving human participants were approved by the ethics committee of Shenzhen Nanshan People's Hospital, in accordance with the ethical standards of Shenzhen University and the 1964 Helsinki declaration and its later amendments, or comparable ethical standards. For this type of study, formal consent is not required.

\section{Antimicrobials susceptibility testing}

Antimicrobial susceptibilities of enterococcus to a several clinical antibiotics, including TEL, ERY, AMP, VAN, TET, DOX, MIN, CIP, NIT, LZD, TED and TEC were tested

with the VITEK 2 system. The minimum inhibitory concentration (MIC) of AMP, VAN, TEL and ERY were identified by the broth macrodilution method according to the CLSI guidelines. As the breakpoint of TEL against enterococci have not been established, we referred to the value of TEL against Staphylococcus aureus (MIC $\leq 1 \mu \mathrm{g} / \mathrm{mL}, 2 \mu \mathrm{g} / \mathrm{mL}, \geq 4 \mu \mathrm{g} / \mathrm{mL}$ ) based on the $2019 \mathrm{CLSI}$ guidelines. We employed five MIC levels for TEL in the antimicrobial susceptibility analysis $\mathrm{MIC} \leq 1,2,4, \geq 8 \mu \mathrm{g} / \mathrm{mL}$.

\section{PCR analyses}

DNA was extracted from all clinical enterococcal isolates in lysis buffer and as templates for PCR according to the manufacturer's instructions (Takara Bio Inc., Japan). All of the microbial DNA served as templates were stored at - $20^{\circ} \mathrm{C}$. PCR analysis was performed to detect ermA, ermB, and erm $C$ genes as described previously. The primer pairs used for PCR amplification were as follows: ermA: sense, 5'- TCTAAAAAGCATGTAAAAGAAA-3' and antisense 5'- CGATACTTTTTGTAGTCCTTC-3'; ermB: sense, 5'-CCGTTTACGAAATTGGAACAGGTAAAGGGC-3' and antisense 5'-GAATCGAGACTTGAGTGTGC-3'; ermC. sense, 5'-GCTAATATTGTTTAAATCGTCAATTCC-3' and antisense 5'- GGATCAGGAAAAGGACATTTTAC -3'.

\section{Multilocus sequence typing}

MLST was performed to analyze the genotypes of the enterococcal isolates. Seven pairs of housekeeping genes: gdh, gyd, pstS, gki, aroE, xpt and $y q i L$ for $E$. faecalis, and atpA, ddl, gdh, purK, gyd, pstS and adk for E. faecium were amplified by PCR. The purified PCR products were sequenced, and results were submitted to the MLST database (http://efaecalis.mlst.net/ and http://efaecium.mlst.net/ ) for comparison, and the sequence types (STs) of enterococci were determined. The primer pairs used for PCR amplification as listed in Table S4 and Table S5 have been described previously [33]. 
As previously described [34], the E. faecalis isolates were diluted $1: 200$ and cultivated in $200 \mu$ l tryptic soy broth (TSB) medium at $37{ }^{\circ} \mathrm{C}$, and inoculated into 96 polystyrene microtiter plates with TSBG (TSB+0.5\% glucose) containing the indicated concentrations of telithromycin. After incubation for 24 hours, biofilm biomasses were stained with crystal violet and stained biofilms were detected with an optical density (OD570). For eradication assays of established biofilms, the $E$. faecalis isolates were cultivated in tryptic soy broth (TSB) medium at $37^{\circ} \mathrm{C}$ for $24 \mathrm{~h}$ to form matured biofilms, and then treated with antimicrobials $(8 \times \mathrm{MIC})$ for $48 \mathrm{~h}$ with the medium replaced daily, the biofilm biomasses were stained and detected. The results were collected from three independent experiments.

\section{Statistical analysis}

Statistical analyses were performed with the SPSS software (version 19.0) and GraphPad Prism software (version 5.0). Results are showed as mean \pm SD. The $p$-value $<0.05$ was regarded as statistically significant.

\section{Abbreviations}

MICs: Minimum inhibitory concentrations; MLST: Multilocus sequence typing; STs: Sequence types; TEL: telithromycin; ERY: erythromycin; AMP: ampicillin; VAN: vancomycin; TET: tetracycline; DOX: doxycycline MIN: minocycline; CIP: ciprofloxacin; NIT: nitrofurantoin; LZD: linezolid; TED: tedizolid; TEC: tetracycline; PCR: Polymerase chain reaction

\section{Declarations}

\section{Ethics approval and consent to participate}

All procedures involving human participants were approved by the ethics committee of Shenzhen Nanshan People's Hospital, in accordance with the ethical standards of Shenzhen University and the 1964 Helsinki declaration and its later amendments, or comparable ethical standards. For this type of study, formal consent is not required.

\section{Consent for publication}

Not Applicable.

\section{Availability of data and materials}

All data supporting the conclusion described in this study are included within the article.

\section{Competing interests}

The authors declare that there is no competing interest.

\section{Funding}

This work was supported by the following grants: the collection of bacterial isolates, antimicrobials susceptibility tests, PCR analyses and MLST analyze were supported by the manipulation from Science, Technology and Innovation Commission of Shenzhen Municipality of key funds (JCYJ20180508162403996) and basic research funds (JCYJ20180302144721183; JCYJ20180302144340004; JCYJ20180302144345028; JCYJ20180302144431923); The inhibition and eradication of E. faecalis biofilms were supported by Sanming Project of Medicine in Shenzhen (No.SMGC201705029) and the Shenzhen Nanshan District Scientific Research Program of the People's Republic of China (No. 2019042, NO.2019051, NO.2019046, NO.2019027, NO.2019040) and provincial medical funds of Guangdong (2018116164215307).

\section{Authors' Contributions}

YX conducted the PCR analyses, MLST analyze, inhibition and eradication of E. faecalis biofilms, drafted the manuscript. JC collected the bacterial isolates,

performed the antimicrobials susceptibility tests and gene manipulation. XS participated in the gene manipulation, MLST analyze, inhibition of $E$. faecalis biofilms. GX, PL participated in the collection of bacterial isolates, MLST analyze, inhibition and eradication of E. faecalis biofilms. ZY, QD participated in the collection of bacterial isolates, inhibition and eradication of E. faecalis biofilms. ZC, JZ designed the study, analyzed the experimental data and revised the manuscript.

all authors have read and approved the manuscript.

\section{Acknowledgements}


The authors would like to thank Yang Wu (Key Laboratory of Medical Molecular Virology of Ministries of Education and Health, School of Basic Medical Science and Institutes of Biomedical Sciences, Shanghai Medical College of Fudan University) for his excellent technical support and suggestions.

\section{References}

1. Mohamed JA, Huang DB: Biofilm formation by enterococci. J Med Microbio/ 2007, 56(Pt 12):1581-1588.

2. Murray BE, Weinstock GM: Enterococci: New Aspects of an Old Organism. Proceedings of the Association of American Physicians 2010, 111(4):328334.

3. Fracp MJR, „, Ms JRE, Culver DH, Gaynes, Robert P., System TNNIS: Nosocomial Infections in Combined Medical-Surgical Intensive Care Units in the United States??? Infection Control \& Hospital Epidemiology 2000, 21(8):510-515.

4. Jones ME, Draghi DC, Thornsberry C, Karlowsky JA, Wenzel RP: Emerging resistance among bacterial pathogens in the intensive care unit - A European and North American Surveillance study (2000-2002). Ann Clin Microbiol Antimicrob 2004, 3(1):14.

5. Jett BD, Huycke MM, Gilmore MS: Virulence of enterococci. 1994, 7(4):462-478.

6. Weng PL, Ramli R, Hamat RA: Antibiotic Susceptibility Patterns, Biofilm Formation and esp Gene among Clinical Enterococci: Is There Any Association? Int J Environ Res Public Health 2019, 16(18).

7. Bhardwaj SB, Mehta M, Sood S, Sharma J: Biofilm Formation by Drug Resistant Enterococci Isolates Obtained from Chronic Periodontitis Patients. J Clin Diagn Res 2017, 11(1):DC01-DC03.

8. Morosini MI, Cercenado E, Ardanuy C, Torres C: [Phenotypic detection of resistance mechanisms in gram-positive bacteria]. Enfermedades Infecciosas Y Microbiología Clínica 2011, 30(6):325-332.

9. Jones R, Low D, Pfaller M: Epidemiologic trends in nosocomial and community-acquired infections due to antibiotic-resistant gram-positive bacteria: the role of streptogramins and other newer compounds. Diagnostic Microbiology \& Infectious Disease 1999, 33(2):101-112.

10. Nguyen M, Chung EP: Telithromycin: The first ketolide antimicrobial. 2005, 27(8):0-1163.

11. Douthwaite S, Champney WS: Structures of ketolides and macrolides determine their mode of interaction with the ribosomal target site. $J$ Antimicrob Chemother 2001, 48(suppl 2):1-8.

12. Vester B, Douthwaite S: Macrolide Resistance Conferred by Base Substitutions in 23S rRNA. Antimicrobial Agents \& Chemotherapy $2001,45(1): 1-12$.

13. Miller WR, Munita JM, Arias CA: Mechanisms of antibiotic resistance in enterococci. Expert Review of Anti-infective Therapy 2014, 12(10):12211236.

14. Westh $\mathrm{H}$, Hougaard DM, Vuust J, Rosdahl VT: Prevalence of erm gene classes in erythromycin-resistant Staphylococcus aureus strains isolated between 1959 and 1988. Antimicrobial Agents \& Chemotherapy 1995, 39(2):369-373.

15. Grit A, Rodloff AC: Drugs of the 21st century: telithromycin (HMR 3647)-the first ketolide. Journal of Antimicrobial Chemotherapy 2003 (3):3.

16. Tatsuma S, Akiko T, Yoshiharu S, Satoshi K, Tsutomu S, Tomoko Y: RImCD-mediated U747 methylation promotes efficient G748 methylation by methyltransferase RImAll in 23S rRNA in Streptococcus pneumoniae; interplay between two rRNA methylations responsible for telithromycin susceptibility. Nucleic Acids Research 2015(18):18.

17. Cetinkaya Y, Falk P, Mayhall CG: Vancomycin-resistant enterococci. Clinical microbiology reviews 2000, 13(4):686-707.

18. Raza T, Ullah SR, Mehmood K, Andleeb S: Vancomycin resistant Enterococci: A brief review. J Pak Med Assoc 2018, 68(5):768-772.

19. Togami K, Chono S, Seki T, Morimoto K: Distribution characteristics of telithromycin, a novel ketolide antimicrobial agent applied for treatment of respiratory infection, in lung epithelial lining fluid and alveolar macrophages. Drug Metab Pharmacokinet 2009, 24(5):411-417.

20. Wolter N, Smith AM, Farrell DJ, Northwood JB, Douthwaite S, Klugman KP: Telithromycin resistance in Streptococcus pneumoniae is conferred by a deletion in the leader sequence of erm(B) that increases rRNA methylation. Antimicrob Agents Chemother 2008, 52(2):435-440.

21. Uzun B, Gungor S, Pektas B, Aksoy Gokmen A, Yula E, Kocal F, Kaya S: [Macrolide-lincosamide-streptogramin B (MLSB) resistance phenotypes in clinical Staphylococcus isolates and investigation of telithromycin activity]. Mikrobiyol Bul 2014, 48(3):469-476.

22. Hiroshige M, Xiang-Hua Y, Mochiyoshi N, Teruhiko T: In vitro and in vivo Antibacterial Activities of Telithromycin. Chemotherapy 2003, $49(1-2): 62-65$.

23. Toledo C, Perez ME, Rocchi M, Gribaudo G, Mangiaterra S, Monterisi A: [Isolation of enterococci species causative of infections and sensitivity to antimicrobial drugs]. Rev Argent Microbiol 2004, 36(1):31-35.

24. Rahim S, Pillai SK, Gold HS, Venkataraman L, Inglima K, Press RA: Linezolid-Resistant, Vancomycin-Resistant Enterococcus faecium Infection in Patients without Prior Exposure to Linezolid. Clinical Infectious Diseases An Official Publication of the Infectious Diseases Society of America 2003, 36(11):e146-e148.

25. Weisblum B, Demohn V: Erythromycin-inducible resistance in Staphylococcus aureus: survey of antibiotic classes involved. J Bacterio/ 1969 , 98(2):447-452.

26. Celik S, Cakirlar FK, Torun MM: Presence of vancomycin, aminoglycosides, and erythromycin resistance genes in enterococci isolated from clinical samples in Turkey. Clin Lab 2014, 60(11):1801-1806.

27. Ruizgarbajosa P, Bonten MJ, Robinson DA, Top J, Nallapareddy SR, Torres C, Coque TM, Cantón R, Baquero F, Murray BE: Multilocus sequence typing scheme for Enterococcus faecalis reveals hospital-adapted genetic complexes in a background of high rates of recombination. 2006, 44(6):2220-2228. 
28. Homan WL, Tribe D, Poznanski S, Li M, Hogg G, Spalburg E, van Embden JDA, Willems RJL: Multilocus Sequence Typing Scheme for Enterococcus faecium. Journal of Clinical Microbiology 2002, 40(9):3548-3548.

29. Ch'ng JH, Chong KKL, Lam LN, Wong JJ, Kline KA: Biofilm-associated infection by enterococci. Nat Rev Microbio/ 2019, 17(2):82-94.

30. Paganelli FL, Willems RJ, Leavis HL: Optimizing future treatment of enterococcal infections: attacking the biofilm? Trends Microbio/ 2012, 20(1):4049.

31. Holmberg A, Rasmussen M: Mature biofilms of Enterococcus faecalis and Enterococcus faecium are highly resistant to antibiotics. Diagn Microbiol Infect Dis 2016, 84(1):19-21.

32. Zheng JX, Tu HP, Sun X, Xu GJ, Chen JW, Deng QW, Yu ZJ, Qu D: In vitro activities of telithromycin against Staphylococcus aureus biofilms compared with azithromycin, clindamycin, vancomycin and daptomycin. J Med Microbiol 2020, 69(1):120-131.

33. Bai B, Hu K, Li H, Yao W, Li D, Chen Z, Cheng H, Zheng J, Pan W, Deng M et al: Effect of tedizolid on clinical Enterococcus isolates: in vitro activity, distribution of virulence factor, resistance genes and multilocus sequence typing. FEMS Microbiol Lett 2018, 365(3).

34. Sun X, Lin ZW, Hu XX, Yao WM, Bai B, Wang HY, Li DY, Chen Z, Cheng H, Pan WG et al: Biofilm formation in erythromycin-resistant Staphylococcus aureus and the relationship with antimicrobial susceptibility and molecular characteristics. Microb Pathog 2018, 124:47-53.

\section{Tables}

Table 1 In vitro antibacterial activity of TEL compared with various antibiotics against $E$. faecalis isolates.

\begin{tabular}{|c|c|c|c|c|c|c|c|c|c|c|c|c|c|c|}
\hline \multirow[t]{2}{*}{ Antibiotic } & \multirow{2}{*}{$\begin{array}{l}\text { No. } \\
\text { isolates }\end{array}$} & \multirow{2}{*}{$\begin{array}{l}\text { Resistance } \\
\text { rate (\%) }\end{array}$} & \multirow{2}{*}{$\begin{array}{l}\text { MIC } \\
(\mu \mathrm{g} / \mathrm{mL}) \\
\text { breakpoints }\end{array}$} & \multirow[t]{2}{*}{ No. } & \multicolumn{6}{|c|}{ TEL MIC $(\mu \mathrm{g} / \mathrm{mL})$} & \multicolumn{4}{|c|}{ ERY MIC $(\mu \mathrm{g} / \mathrm{mL})$} \\
\hline & & & & & $\leq 0.5$ & 1 & 2 & 4 & $\geq 8$ & $\mathrm{MIC}_{50} / \mathrm{MIC}_{90}$ & $\leq 0.5$ & $\begin{array}{l}1- \\
4\end{array}$ & $\geq 8$ & $\begin{array}{l}\mathrm{MIC}_{50} \\
/ \mathrm{MIC}_{90}\end{array}$ \\
\hline Total & 280 & - & - & - & 116 & 7 & 27 & 97 & 33 & $2 / 8$ & 10 & 58 & 212 & $>256 />256$ \\
\hline \multirow[t]{2}{*}{ Ampicillin } & 275 & 0.4 & $\leq 8$ & 274 & 130 & 6 & 26 & 83 & 29 & $2 / 8$ & 8 & 55 & 211 & $>256 />256$ \\
\hline & & & $\geq 16$ & 1 & 0 & 0 & 0 & 1 & 0 & $4 / 4$ & 0 & 0 & 1 & - \\
\hline \multirow[t]{3}{*}{ Vancomycin } & 280 & 0 & $\leq 4$ & 278 & 115 & 7 & 27 & 96 & 33 & $2 / 8$ & 10 & 58 & 210 & $>256 />256$ \\
\hline & & & $8-16$ & 2 & 1 & 0 & 0 & 1 & 0 & $0.125 / 4$ & 0 & 0 & 2 & $>256 />256$ \\
\hline & & & $\geq 32$ & 0 & 0 & 0 & 0 & 0 & 0 & - & 0 & 0 & 0 & - \\
\hline \multirow[t]{3}{*}{ Tetracycline } & 276 & 83.7 & $\leq 4$ & 39 & 33 & 3 & 1 & 2 & 0 & $0.625 / 1$ & 7 & 17 & 15 & $4 />256$ \\
\hline & & & 8 & 6 & 3 & 0 & 0 & 2 & 1 & $0.625 / 4$ & 1 & 1 & 4 & $128 />256$ \\
\hline & & & $\geq 16$ & 231 & 79 & 4 & 25 & 91 & 32 & $4 / 8$ & 2 & 39 & 190 & $>256 />256$ \\
\hline \multirow[t]{3}{*}{ Doxycycline } & 280 & 78.9 & $\leq 4$ & 40 & 36 & 1 & 2 & 1 & 0 & $0.0625 / 0.125$ & 7 & 19 & 14 & $2 />256$ \\
\hline & & & 8 & 19 & 7 & 2 & 3 & 6 & 1 & $2 / 4$ & 1 & 2 & 16 & $128 />256$ \\
\hline & & & $\geq 16$ & 221 & 73 & 4 & 22 & 90 & 32 & $4 / 8$ & 2 & 37 & 182 & $>256 />256$ \\
\hline \multirow[t]{3}{*}{ Minocycline } & 280 & 73.2 & $\leq 4$ & 43 & 37 & 2 & 2 & 2 & 0 & $0.625 / 2$ & 7 & 21 & 15 & $2 />256$ \\
\hline & & & 8 & 32 & 11 & 0 & 9 & 9 & 3 & $2 / 4$ & 1 & 5 & 26 & $>256 />256$ \\
\hline & & & $\geq 16$ & 205 & 68 & 5 & 16 & 86 & 30 & $4 / 8$ & 2 & 32 & 171 & $>256 />256$ \\
\hline \multirow[t]{3}{*}{ Ciprofloxacin } & 252 & 26.6 & $\leq 1$ & 151 & 68 & 3 & 11 & 51 & 18 & $2 / 8$ & 5 & 25 & 121 & $>256 />256$ \\
\hline & & & 2 & 34 & 22 & 1 & 2 & 8 & 1 & $0.125 / 4$ & 1 & 12 & 21 & $128 />256$ \\
\hline & & & $\geq 4$ & 67 & 24 & 3 & 14 & 20 & 6 & $2 / 8$ & 2 & 12 & 53 & $>256 />256$ \\
\hline \multirow[t]{3}{*}{ Nitrofurantoin } & 254 & 1.2 & $\leq 32$ & 247 & 102 & 6 & 26 & 84 & 29 & $2 / 8$ & 9 & 52 & 186 & $>256 />256$ \\
\hline & & & 64 & 4 & 2 & 0 & 0 & 2 & 0 & $0.5 / 4$ & 0 & 0 & 4 & $>256 />256$ \\
\hline & & & $\geq 128$ & 3 & 3 & 0 & 0 & 0 & 0 & $0.03 / 0.25$ & 0 & 2 & 1 & $4 />128$ \\
\hline \multirow[t]{3}{*}{ Linezolid } & 280 & 5.4 & $\leq 2$ & 214 & 87 & 6 & 21 & 75 & 25 & $2 / 8$ & 9 & 44 & 161 & $>256 />256$ \\
\hline & & & 4 & 51 & 23 & 1 & 6 & 17 & 4 & $2 / 4$ & 1 & 11 & 39 & $>256 />256$ \\
\hline & & & $\geq 8$ & 15 & 6 & 0 & 0 & 5 & 4 & $4 / 8$ & 0 & 3 & 12 & $>256 />256$ \\
\hline Tedizolid & 279 & - & $\leq 0.5$ & 239 & 97 & 7 & 25 & 81 & 29 & $2 / 8$ & 9 & 45 & 185 & $>256 />256$ \\
\hline
\end{tabular}


Note: MIC, minimum inhibitory concentration; TEL, telithromycin; ERY, erythromycin;

Table 2 In vitro antibacterial activity of TEL compared with various antibiotics against $E$. faecium isolates.

\begin{tabular}{|c|c|c|c|c|c|c|c|c|c|c|c|c|c|c|}
\hline \multirow[t]{2}{*}{ Antibiotic } & \multirow{2}{*}{$\begin{array}{l}\text { No. } \\
\text { isolates }\end{array}$} & \multirow{2}{*}{$\begin{array}{l}\text { Resistance } \\
\text { rate (\%) }\end{array}$} & \multirow{2}{*}{$\begin{array}{l}\text { MIC } \\
(\mu \mathrm{g} / \mathrm{mL}) \\
\text { breakpoints }\end{array}$} & \multirow[t]{2}{*}{ No. } & \multicolumn{5}{|c|}{ TEL MIC $(\mu \mathrm{g} / \mathrm{mL})$} & \multicolumn{5}{|c|}{ ERY MIC $(\mu \mathrm{g} / \mathrm{mL})$} \\
\hline & & & & & $\leq 0.5$ & 1 & 2 & 4 & $\geq 8$ & $\begin{array}{l}\mathrm{MIC}_{50} \\
/ \mathrm{MIC}_{90}\end{array}$ & $\leq 0.5$ & $\begin{array}{l}1- \\
4\end{array}$ & $\geq 8$ & $\mathrm{MIC}_{50} / \mathrm{MIC}_{90}$ \\
\hline Total & 122 & - & - & - & 25 & 2 & 12 & 34 & 49 & $4 / 8$ & 6 & 12 & 104 & $>256 />256$ \\
\hline \multirow[t]{2}{*}{ Ampicillin } & 112 & 87.5 & $\leq 8$ & 14 & 11 & 0 & 0 & 3 & 0 & $0.063 / 4$ & 1 & 6 & 7 & $8 />128$ \\
\hline & & & $\geq 16$ & 98 & 13 & 2 & 12 & 29 & 42 & $4 / 8$ & 5 & 6 & 87 & $>128 />256$ \\
\hline \multirow[t]{3}{*}{ Vancomycin } & 120 & 0.0 & $\leq 4$ & 116 & 23 & 2 & 12 & 31 & 48 & $4 / 8$ & 6 & 11 & 99 & $128 />256$ \\
\hline & & & $8-16$ & 4 & 2 & 0 & 0 & 2 & 0 & $0.063 / 4$ & 0 & 1 & 3 & $8 />256$ \\
\hline & & & $\geq 32$ & 0 & 0 & 0 & 0 & 0 & 0 & - & 0 & 0 & 0 & - \\
\hline \multirow[t]{3}{*}{ Teicoplanin } & 115 & 0.9 & $\leq 8$ & 114 & 24 & 2 & 12 & 33 & 43 & $4 / 8$ & 6 & 12 & 96 & $>128 />256$ \\
\hline & & & 16 & 0 & 0 & 0 & 0 & 0 & 0 & - & 0 & 0 & 0 & - \\
\hline & & & $\geq 32$ & 1 & 0 & 0 & 0 & 0 & 1 & 8 & 0 & 0 & 1 & $>256 />256$ \\
\hline \multirow[t]{3}{*}{ Tetracycline } & 119 & 46.2 & $\leq 4$ & 45 & 11 & 1 & 1 & 16 & 16 & $4 / 8$ & 1 & 6 & 38 & $>128 />256$ \\
\hline & & & 8 & 19 & 3 & 0 & 1 & 3 & 12 & $8 / 8$ & 2 & 0 & 17 & $>256 />256$ \\
\hline & & & $\geq 16$ & 55 & 10 & 1 & 10 & 14 & 20 & $4 / 8$ & 3 & 5 & 47 & $>256 />256$ \\
\hline \multirow[t]{3}{*}{ Doxycycline } & 120 & 38.3 & $\leq 4$ & 62 & 15 & 1 & 4 & 22 & 20 & $4 / 8$ & 4 & 8 & 50 & $>128 />256$ \\
\hline & & & 8 & 12 & 1 & 0 & 4 & 1 & 6 & $4 / 8$ & 0 & 0 & 12 & $>256 />256$ \\
\hline & & & $\geq 16$ & 46 & 9 & 1 & 4 & 8 & 24 & $8 / 8$ & 2 & 4 & 40 & $>128 />256$ \\
\hline \multirow[t]{3}{*}{ Minocycline } & 120 & 27.5 & $\leq 4$ & 66 & 16 & 1 & 4 & 22 & 23 & $4 / 8$ & 4 & 8 & 54 & $>128 />256$ \\
\hline & & & 8 & 21 & 3 & 1 & 6 & 4 & 7 & $2 / 8$ & 1 & 1 & 19 & $>256 />256$ \\
\hline & & & $\geq 16$ & 33 & 6 & 0 & 2 & 7 & 18 & $8 / 8$ & 1 & 3 & 29 & $>128 />256$ \\
\hline \multirow[t]{3}{*}{ Ciprofloxacin } & 113 & 9.7 & $\leq 1$ & 29 & 10 & 1 & 7 & 8 & 3 & $2 / 4$ & 1 & 5 & 23 & $>256 />256$ \\
\hline & & & 2 & 4 & 1 & 0 & 0 & 1 & 2 & $4 / 8$ & 1 & 0 & 3 & $>256 />256$ \\
\hline & & & $\geq 4$ & 80 & 13 & 1 & 5 & 24 & 37 & $4 / 8$ & 3 & 7 & 70 & $>128 />256$ \\
\hline \multirow[t]{3}{*}{ Nitrofurantoin } & 114 & 57.0 & $\leq 32$ & 11 & 2 & 0 & 3 & 2 & 4 & $4 / 8$ & 1 & 1 & 9 & $>256 />256$ \\
\hline & & & 64 & 38 & 6 & 1 & 5 & 12 & 14 & $4 / 8$ & 1 & 4 & 33 & $>256 />256$ \\
\hline & & & $\geq 128$ & 65 & 16 & 1 & 4 & 18 & 26 & $4 / 8$ & 4 & 7 & 54 & $>128 />256$ \\
\hline \multirow[t]{3}{*}{ Linezolid } & 122 & 2.4 & $\leq 2$ & 116 & 22 & 2 & 12 & 32 & 48 & $4 / 8$ & 6 & 10 & 100 & $>128 />256$ \\
\hline & & & 4 & 3 & 2 & 0 & 0 & 1 & 0 & $0.063 / 4$ & 0 & 1 & 2 & $8 />256$ \\
\hline & & & $\geq 8$ & 3 & 1 & 0 & 0 & 1 & 1 & $4 / 8$ & 0 & 1 & 2 & $>256 />256$ \\
\hline
\end{tabular}

Note: MIC, minimum inhibitory concentration; TEL, telithromycin; ERY, erythromycin;

Table 3 In vitro activity of TEL against $E$. faecalis isolates with ERY-specific resistant genes. 


\begin{tabular}{|c|c|c|c|c|c|c|c|c|c|c|c|c|}
\hline \multirow{2}{*}{\multicolumn{2}{|c|}{$\begin{array}{l}\text { Erythromycin } \\
\text { resistance genes }\end{array}$}} & \multirow[t]{2}{*}{ No. } & \multicolumn{6}{|c|}{ TEL MIC $(\mu \mathrm{g} / \mathrm{mL})$} & \multicolumn{4}{|c|}{ ERY MIC $(\mu \mathrm{g} / \mathrm{mL})$} \\
\hline & & & $\leq 0.5$ & 1 & 2 & 4 & $\geq 8$ & MIC50 /MIC90 & $\leq 0.5$ & $1-4$ & $\geq 8$ & MIC50 /MIC90 \\
\hline Total & & 280 & 116 & 7 & 27 & 97 & 33 & $2 / 8$ & 10 & 58 & 212 & $>256 />256$ \\
\hline \multirow[t]{2}{*}{ ermA } & + & 11 & 1 & 0 & 0 & 6 & 4 & $4 / 8$ & 0 & 0 & 11 & $>256 />256$ \\
\hline & - & 269 & 115 & 7 & 27 & 91 & 29 & $2 / 8$ & 10 & 58 & 201 & $>256 />256$ \\
\hline \multirow[t]{2}{*}{$e r m B$} & + & 188 & 39 & 4 & 23 & 92 & 30 & $4 / 8$ & 1 & 8 & 179 & $>256 />256$ \\
\hline & - & 92 & 77 & 3 & 4 & 5 & 3 & $0.625 / 2$ & 9 & 50 & 33 & $2 />256$ \\
\hline \multirow[t]{2}{*}{ ermc } & + & 0 & 0 & 0 & 0 & 0 & 0 & - & 0 & 0 & 0 & - \\
\hline & - & 280 & 116 & 7 & 27 & 97 & 33 & $2 / 8$ & 10 & 58 & 212 & $>256 />256$ \\
\hline
\end{tabular}

Note: MIC, minimum inhibitory concentration; TEL, telithromycin; ERY, erythromycin; +, positive; -, negative;

Table 4 In vitro activity of TEL against E. faecium isolates with ERY-specific resistant genes

\begin{tabular}{|c|c|c|c|c|c|c|c|c|c|c|c|c|}
\hline \multirow{2}{*}{\multicolumn{2}{|c|}{$\begin{array}{l}\text { Erythromycin } \\
\text { resistance genes }\end{array}$}} & \multirow[t]{2}{*}{ No. } & \multicolumn{6}{|c|}{ TEL MIC $(\mu \mathrm{g} / \mathrm{mL})$} & \multicolumn{4}{|c|}{ ERY MIC $(\mu \mathrm{g} / \mathrm{mL})$} \\
\hline & & & $\leq 0.5$ & 1 & 2 & 4 & $\geq 8$ & MIC50 /MIC90 & $\leq 0.5$ & $1-4$ & $\geq 8$ & MIC50 /MIC9C \\
\hline Total & & 122 & 25 & 2 & 12 & 34 & 49 & $4 / 8$ & 6 & 12 & 104 & $\geq 0.25-256$ \\
\hline \multirow[t]{2}{*}{ ermA } & + & 12 & 2 & 0 & 2 & 3 & 5 & $4 / 8$ & 0 & 2 & 10 & $128 />256$ \\
\hline & - & 110 & 23 & 2 & 10 & 31 & 44 & $4 / 8$ & 6 & 10 & 94 & $>128 />256$ \\
\hline \multirow[t]{2}{*}{$e r m B$} & + & 40 & 2 & 0 & 3 & 21 & 14 & $4 / 8$ & 0 & 2 & 38 & $128 />256$ \\
\hline & - & 82 & 23 & 2 & 9 & 13 & 35 & $4 / 8$ & 6 & 10 & 66 & $>128 />256$ \\
\hline \multirow[t]{2}{*}{ ermc } & + & 56 & 5 & 2 & 7 & 10 & 32 & $8 / 8$ & 0 & 1 & 55 & $>256 />256$ \\
\hline & - & 66 & 20 & 0 & 5 & 24 & 17 & $4 / 8$ & 6 & 11 & 49 & $>128 />256$ \\
\hline
\end{tabular}

Note: MIC, minimum inhibitory concentration; TEL, telithromycin; ERY, erythromycin; +, positive; -, negative

Table 5 TEL MIC values of predominant STs in E. faecalis isolates with carrying erm $B$ gene

\begin{tabular}{|c|c|c|c|c|c|c|c|c|c|c|c|c|}
\hline \multirow[b]{2}{*}{ ST } & \multirow[t]{2}{*}{ No. (\%) } & \multicolumn{6}{|c|}{ TEL MIC $(\mu \mathrm{g} / \mathrm{mL})$} & \multicolumn{4}{|c|}{ ERY MIC $(\mu \mathrm{g} / \mathrm{mL})$} & \multirow[t]{2}{*}{$e r m B$} \\
\hline & & $\leq 0.5$ & 1 & 2 & 4 & $\geq 8$ & $\mathrm{MIC}_{50} / \mathrm{MIC}_{90}$ & $\leq 0.5$ & $1-4$ & $\geq 8$ & $\mathrm{MIC}_{50} / \mathrm{MIC}_{90}$ & \\
\hline ST16 & 78 (27.9) & 18 & 3 & 9 & 36 & 12 & $4 / 8$ & 0 & 6 & 72 & $>256 />256$ & 67 \\
\hline ST30 & $8(2.9)$ & 7 & 0 & 1 & 0 & 0 & $0.125 / 2$ & 0 & 2 & 6 & $128 />256$ & 6 \\
\hline ST179 & $72(25.7)$ & 14 & 0 & 5 & 39 & 14 & $4 / 8$ & 2 & 7 & 63 & $>256 />256$ & 62 \\
\hline
\end{tabular}

Note: MIC, minimum inhibitory concentration; TEL, telithromycin; ERY, erythromycin

Table 6 TEL MIC values of predominant STs in E. faecium isolates with carrying erm B and erm C genes.

\begin{tabular}{|c|c|c|c|c|c|c|c|c|c|c|c|c|c|}
\hline \multirow[b]{2}{*}{ ST } & \multirow[t]{2}{*}{ No. (\%) } & \multicolumn{6}{|c|}{ TEL MIC $(\mu \mathrm{g} / \mathrm{mL})$} & \multicolumn{4}{|c|}{ ERY MIC $(\mu \mathrm{g} / \mathrm{mL})$} & \multirow[t]{2}{*}{$e r m B$} & \multirow[t]{2}{*}{ ermC } \\
\hline & & $\leq 0.5$ & 1 & 2 & 4 & $\geq 8$ & $\mathrm{MIC}_{50} / \mathrm{MIC}_{90}$ & $\leq 0.5$ & $1-4$ & $\geq 8$ & $\mathrm{MIC}_{50} / \mathrm{MIC}_{90}$ & & \\
\hline ST18 & 18 (12.3) & 3 & 0 & 0 & 0 & 15 & $8 / 8$ & 0 & 1 & 17 & $>256 />256$ & 1 & 17 \\
\hline ST78 & $26(21.3)$ & 4 & 1 & 0 & 9 & 12 & $4 / 8$ & 3 & 1 & 22 & $128 />256$ & 16 & 3 \\
\hline ST80 & $7(5.7)$ & 0 & 0 & 1 & 3 & 3 & $4 / 8$ & 0 & 0 & 7 & $>128 />128$ & 4 & 0 \\
\hline
\end{tabular}


Figures
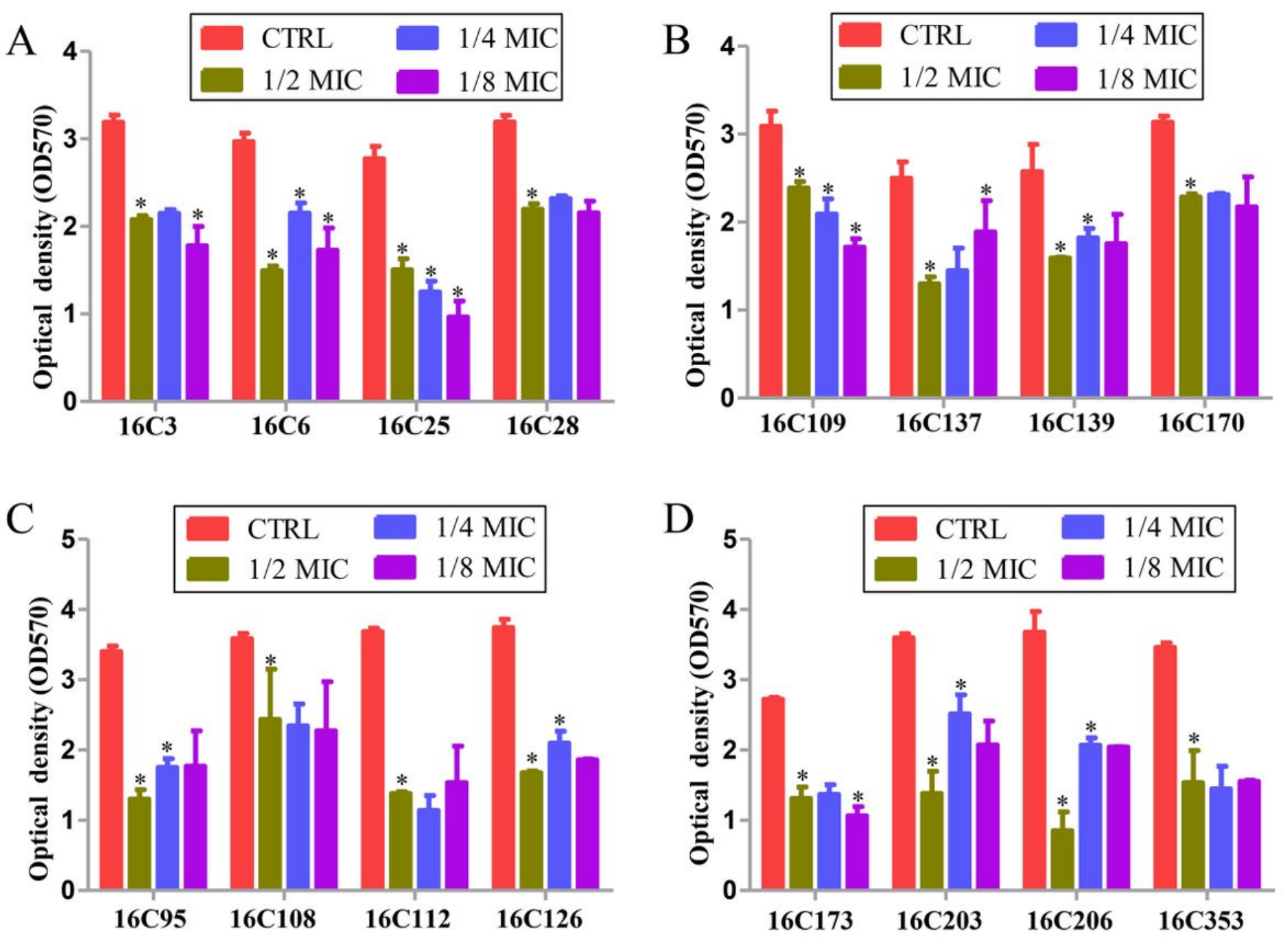

Figure 1

Telithromycin inhibiting the biofilm formation of $16 \mathrm{E}$. faecalis isolates. (A) and (B) The four isolates of per group were treated with TEL at $1 / 2 \times, 1 / 4 \times$ and $1 / 8 \times$ MICs, respectively. All of the 8 isolates with the TEL MIC $=8 \mu \mathrm{g} / \mathrm{mL}$. (C) and (D) The four isolates of per group were treated with TEL at $1 / 2 \times$, $1 / 4 \times$ and $1 / 8 \times$ MICs, respectively. All of the 8 isolates with the TEL MIC $\leq 0.5 \mu \mathrm{g} / \mathrm{mL}$. The data presented was the average of three independent experiments (mean $\pm \mathrm{SD}$ ). Statistical significance between groups was calculated by Student's t-test. CTRL, control group; *Compared to CTRL, $P<0.05$. 

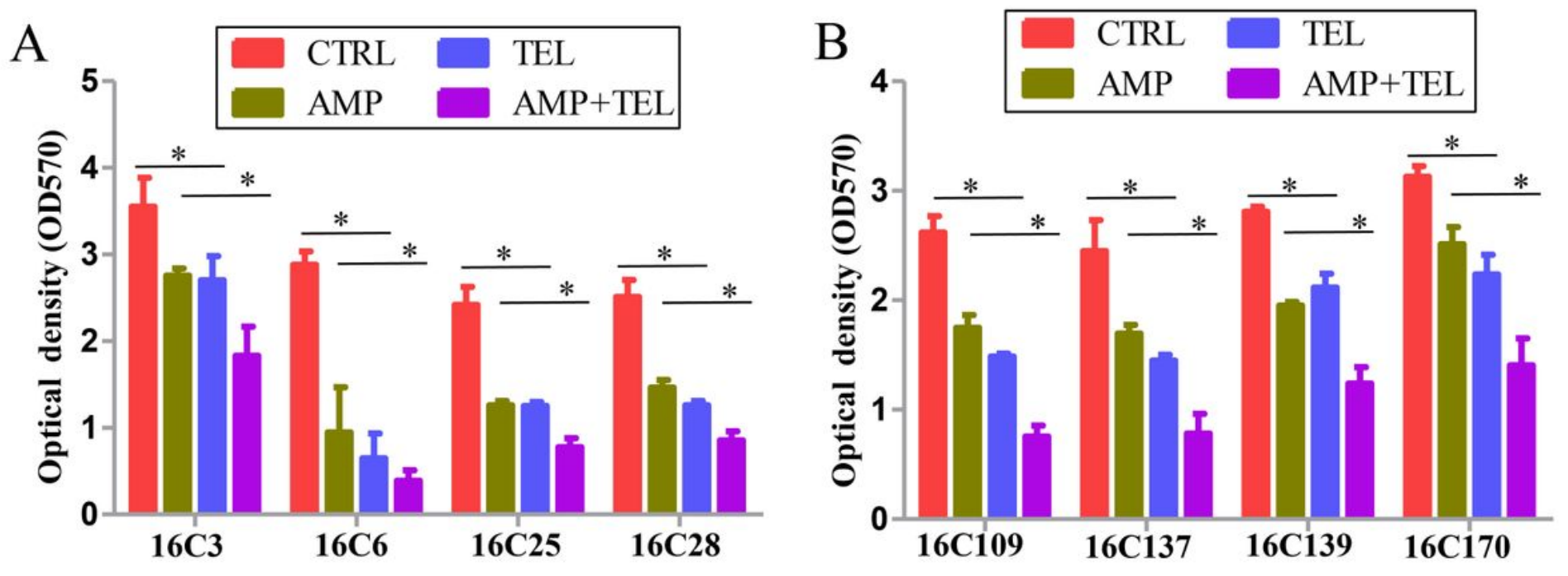

Figure 2

Telithromycin alone or combined with ampicillin eradicating the established biofilms of $8 \mathrm{E}$. faecalis isolates. The total of 8 isolates formed mature biofilms for $24 \mathrm{~h}$, then treated with TEL, or combined with AMP at $8 \times$ MICs for $48 \mathrm{~h}$. The data presented was the average of three independent experiments (mean $\pm \mathrm{SD}$ ). Statistical significance between groups was calculated by Student's t-test. CTRL, control group; TEL, telithromycin; AMP, ampicillin; ${ }^{*} \mathrm{P}<0.05$.

\section{Supplementary Files}

This is a list of supplementary files associated with this preprint. Click to download.

- FigS1.tif

- FigS2.tif

- Supplementarytables2020.4.20.docx 